\title{
A influência da ocupação profissional na flexibilidade global e nas amplitudes angulares dos membros inferiores e da lombar
}

\author{
The influence of occupation on overall flexibility \\ and lower limb and lumbar range of motion
}

\author{
Isabel Camargo Neves Sacco \\ Sandra Aliberti \\ Bergson Weber Cabral Queiroz \\ Denise Pripas \\ Isadora Kieling \\ Aline Aiako Kimura \\ Adriana Elisa Sellmer \\ Renan Amaro Malvestio \\ Marcel Tomonori Sera
}

1 Universidade de São Paulo. Faculdade de Medicina. Departamento de Fisioterapia, Fonoaudiologia e Terapia Ocupacional. Laboratório de Biomecânica do Movimento e Postura Humana. São Paulo, SP. Brasil.

Recebido em 31/10/07 Aprovado em 11/04/08

\begin{abstract}
Resumo - O desenvolvimento tecnológico no ambiente de trabalho gerou um aumento no tempo em que os indivíduos permanecem sentados enquanto trabalham em escritórios. Isto pode estar influenciando negativamente capacidades físicas inatas como a flexibilidade da cadeia posterior do corpo, porém poucos são os estudos que avaliaram a influência do trabalho nesta capacidade física. Esta avaliação pode contribuir para intervenção precoce e paralela à atividade ocupacional, prevenindo disfunções musculoesqueléticas. O objetivo foi verificar se a atividade profissional determina alguma modificação na flexibilidade global da cadeia posterior na postura de flexão do tronco e analisar os segmentos corporais contribuidores desta modificação. Participaram do estudo 24 mulheres saudáveis e sedentárias, entre 18 e 55 anos, que trabalhavam em manutenção $(n=13)$ e em escritório $(n=11)$. Foram avaliados o teste do terceiro dedo ao solo (fita métrica) e os ângulos articulares tíbio-társico, do joelho, do quadril, da lombar e a técnica de Chaffin Modificada por meio da fotogrametria (software SAPo) na postura de flexão do tronco. Os grupos foram comparados por meio de teste $t(\alpha=5 \%)$. Mulheres que trabalham em escritório mostraram maiores distâncias do terceiro dedo ao solo $(p=0,0518)$ e técnica de Chaffin Modificada significativamente menor . ( $p=0,0134)$, enquanto os ângulos tíbio- tarsico e do quadril mostraram valores marginalmente maiores nestas mulheres ( $p=0,0609$ e $p=0,0713$, respectivamente). Mulheres que trabalham predominantemente na posição sentada apresentam menor flexibilidade global da cadeia posterior quando comparadas com as que realizam um trabalho que exige flexão cíclica do tronco. Sugere-se que os ângulos tíbio-tarsico e quadril, em conjunto, são os segmentos corporais que contribuem para esta menor flexibilidade.

Palavras-chave: Flexibilidade; Postura; Amplitude de movimento articular.
\end{abstract}

Abstract - The technological development in the working environment has prolonged sitting time for office employees. This prolonged sitting time may negatively influence innate physical capacities such as flexibility of the posterior chain; however, few studies have evaluated the influence of occupation on this physical capacity. This assessment might contribute to establish an early intervention approach, in parallel to the occupational activity, in order to prevent musculoskeletal dysfunctions. The aim of this study was to determine whether occupational activity influences posterior chain muscle flexibility during trunk-flexed posture and to analyze the body segments that are responsible for any flexibility changes. Twenty-four healthy women aged 18 to 55 years, who worked in cleaning and maintenance $(n=13)$ and in offices $(n=11)$, participated in the study. The following measurements were obtained to evaluate trunk flexion: fingertip-to-floor distance test (measuring tape), tibiotarsal angle, knee extension/flexion angle, hip angle, lumbar angle, and modified Chaffin technique (digital photogrammetry using the SAPo software). The groups were compared using the independent $t$-test. Women working in offices presented the worst fingertip-to-floor test result $(p=0.0518)$ and a lower modified Chaffin technique value ( $p=0.0134)$, whereas their tibiotarsal and hip angles were marginally greater $(p=0.0609$ and $p=0.0713$, respectively). Women who mainly work seated presented lower overall flexibility of the posterior chain muscles than women who perform occupations that require cyclic flexion of the trunk. The tibiotarsal and hip angles seem to be the body segments responsible for this reduction in flexibility.

Key words: Flexibility; Posture; Range of joint motion. 


\section{INTRODUÇÃO}

A maioria dos adultos passa metade do tempo em que estão acordados em seu local de trabalho1. O desenvolvimento tecnológico deste ambiente tem gerado um aumento no tempo em que os trabalhadores permanecem na posição sentada enquanto executam suas atividades em escritórios ${ }^{2,3}$. Este fato pode estar influenciando negativamente capacidades físicas inatas como a flexibilidade da cadeia posterior do corpo. Por outro lado, indivíduos que são ativos fisicamente durante o trabalho, realizando freqüentemente movimentos de flexão do tronco - trabalhadores de manutenção e limpeza, por exemplo - podem apresentar uma maior flexibilidade quando comparados aos trabalhadores que permanecem sentados durante sua atividade ocupacional ${ }^{4}$.

A flexibilidade é a capacidade física responsável pela execução de um movimento de amplitude angular máxima ${ }^{5}$ por uma articulação ou conjunto de articulações, dentro dos limites fisiológicos, sem o risco de ocorrerem lesões ${ }^{6}$. Esta capacidade está, principalmente, relacionada aos hábitos posturais, elasticidade dos músculos e plasticidade dos ligamentos, tendões e cápsulas articulares. Os hábitos posturais, por sua vez podem estar sendo influenciados pelo tipo de atividade ocupacional que o indivíduo realiza. A importância da flexibilidade tem sido destacada nos movimentos diários para melhora da qualidade dos mesmos ${ }^{7}$ ou, ainda, na prevenção de lesões ${ }^{8}$. Apesar de não estar estabelecido o mecanismo pelo qual a flexibilidade contribui na prevenção de lesões, alguns estudos tem apontado a diminuição da flexibilidade como fator de risco para patologias degenerativas, como a lombalgia ${ }^{9,10}$.

Poucos são os estudos que avaliam as características da atividade física realizada durante o trabalho ${ }^{11,12}$ e sua influência nas capacidades físicas dos indivíduos ${ }^{4}$. Estes estudos são relevantes por possibilitar um diagnóstico do tipo de atividade física que deve ser encorajada para grupos específicos de trabalhadores.

$\mathrm{Na}$ literatura, estabelece-se uma relação entre um maior nível de atividade física no trabalho e um melhor condicionamento cardiorrespiratório ${ }^{13}$, força manual e resistência de musculatura de tronco ${ }^{14}$.

No que diz respeito especificamente à flexibilidade, alguns autores concluíram que trabalhadores que realizam funções mais ativas fisicamente no trabalho apresentam uma maior flexibilidade do que os que realizam trabalhos caracterizados como sedentários ${ }^{15,16}$. No entanto, além de escassas, estas pesquisas não abordam uma atividade profissional específica.

Milosavljevic et al. ${ }^{4}$ encontraram um aumento da amplitude de flexão de quadril em tosquiadores de ovelhas, os quais necessitam curvar-se ciclicamente para a execução de suas atividades diárias, demonstrando, assim, a influência da atividade ocupacional na flexibilidade articular. No entanto, essa influência adaptativa ainda não foi estudada em outras profissões. ${ }^{4}$.

Diante do contexto discutido anteriormente, tem-se por objetivo verificar se a atividade profissional determina alguma modificação na flexibilidade global da cadeia posterior na postura de flexão do tronco e analisar os segmentos corporais potencialmente contribuidores desta modificação.

\section{PROCEDIMENTOS METODOLÓGICOS}

Este estudo teve delineamento de pesquisa observacional e transversal, com amostragem intencional. Os critérios de inclusão foram indivíduos saudáveis, do sexo feminino, com idade entre 18 e 55 anos, divididos em um primeiro grupo de voluntárias que trabalhassem em manutenção e limpeza e um segundo grupo composto de voluntárias que exercessem sua atividade profissional em escritório. As voluntárias de ambos os grupos deveriam ser sedentárias e pareadas em massa, estatura e idade. Os critérios de exclusão foram assimetrias posturais importantes e a ocorrência de episódios de dor nos membros inferiores e coluna lombar nos últimos três meses ${ }^{17-19}$. A partir destes critérios, foram excluídas três voluntárias (uma do grupo escritório e duas do grupo manutenção) por apresentarem dor na coluna lombar.

Para a realização do estudo, foi solicitado que os sujeitos assinassem o termo de consentimento informado e aprovado pela comissão de ética local (número 1237/05).

Os sujeitos foram submetidos a um protocolo composto de sete etapas, quais sejam: (1) Entrevista pessoal, (2) Aquisição dos dados antropométricos, (3) Avaliação Postural, (4) Habituação à postura de flexão do tronco, (5) Marcação de referências ósseas com etiquetas auto-adesivas,( 6) Fotografia digital, em posição 


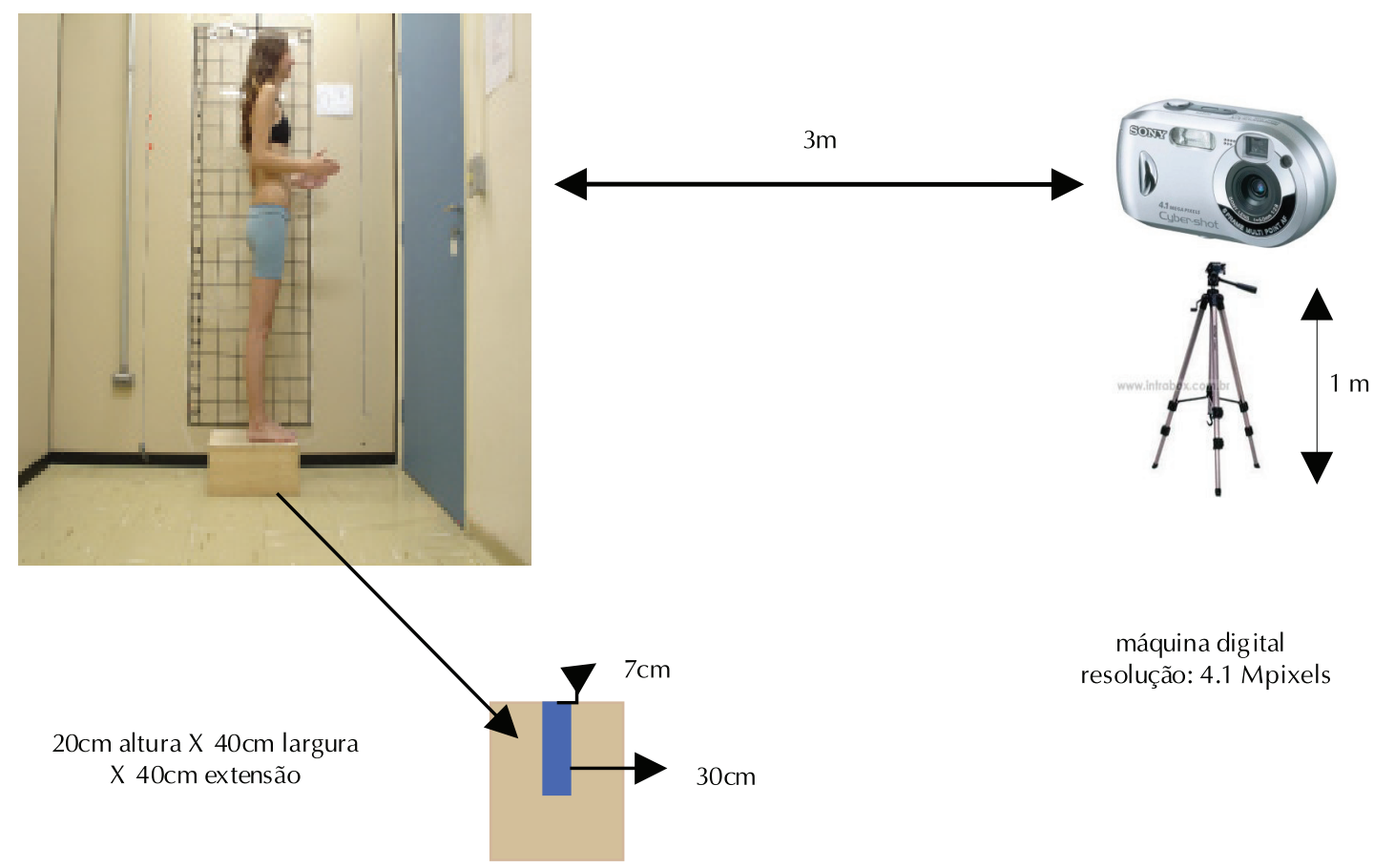

Figura 1. Esquema do setup experimental.

ortostática no plano sagital e (7) Realização do teste do $3^{\circ}$ dedo ao solo com realização de uma segunda fotografia digital.

Nas primeiras duas etapas, foram coletados os dados pessoais e antropométricos dos sujeitos. $\mathrm{Na}$ terceira etapa, foi realizada uma avaliação postural observacional por uma fisioterapeuta experiente, segundo o protocolo de Kendall ${ }^{20}$, a fim de se aplicarem os critérios de exclusão.

Na quarta etapa, foi feita uma habituação dos sujeitos à postura do teste terceiro dedo ao solo. Foi solicitado que eles levassem suas mãos em direção aos pés com os cotovelos estendidos, fletindo o tronco, mantendo os joelhos estendidos, cabeça e ombros relaxados, por três vezes seguidas.

Durante a quinta etapa, pontos anatômicos foram palpados por um mesmo examinador e em seguida, marcados com etiquetas auto-adesivas vermelhas da marca Pimaco, de 0,9cm de diâmetro e hastes cilíndricas plásticas amarelas, de $3,0 \mathrm{~cm}$ de comprimento, presas por fita adesiva dupla face nos pontos anatômicos de difícil visualização por etiquetas. Os pontos anatômicos utilizados foram: maléolo lateral, epicôndilo lateral do fêmur, trocânter maior do fêmur, espinha ilíaca póstero-superior, espinha ilíaca póstero-inferior, espinha ilíaca ântero-superior, centro lateral da articulação do ombro.

$\mathrm{Na}$ sexta etapa, foi obtida uma foto digital no plano perpendicular à máquina, posiciona- da sobre um tripé nivelado e sagital ao corpo com os sujeitos em posição ortostática. Os registros fotográficos obtidos com resolução de 1600X1200 pixels em uma máquina Sony (4.1 megapixels). A máquina permaneceu à 3 metros de distância do sujeito, enquanto o tripé foi posicionado a uma altura de 1,0 metro do chão. Os sujeitos foram posicionados sobre um banco $(20 \mathrm{~cm}$ de altura x $40 \mathrm{~cm}$ de largura e 40 cm de extensão) à $15 \mathrm{~cm}$ de distância da parede. Foi colocado um retângulo de etil vinil acetato (7,0 cm de largura por $30 \mathrm{~cm}$ de extensão) para padronização da distância entre os pés dos voluntários que trajavam biquíni ou sunga em uma sala reservada, bem iluminada e de fundo não reflexivo (figura 1). Além disto, foram posicionados dois fios de prumo, um de cada lado do banco de posicionamento. Estes prumos estavam pendurados no teto e ultrapassavam os pés dos sujeitos posicionados.

Durante a sétima etapa, com as voluntárias ainda posicionadas sobre o banco, foi realizado o teste do $3^{\circ}$ dedo ao solo, solicitando que repetissem a postura descrita na habituação. Após o posicionamento, foi medida - sempre pelo mesmo avaliador - a distância entre o $3^{\circ}$ dedo e o solo por meio de uma fita métrica e em seguida foi obtida uma segunda foto digital na postura do teste.

$O$ teste $3^{\circ}$ dedo ao solo tem sido usado para avaliar a flexibilidade da cadeia posterior 
Tabela 1. Ângulos articulares obtidos por meio das fotografias digitais.

\begin{tabular}{ll}
\hline Variáveis & Descrição da variável \\
\hline Ângulo Tíbio-Társico & $\begin{array}{l}\text { Ângulo entre o segmento de reta paralelo ao solo que passa pelo maléolo lateral e o } \\
\text { segmento de reta que passa pelo maléolo lateral até côndilo femoral lateral, com vérti- } \\
\text { ce no maléolo. } \\
\text { Ângulo entre o segmento de reta que passa pelo trocânter maior do fêmur até o côndilo } \\
\text { femoral lateral e outro segmento que passa pelo trocânter maior até a projeção da espi- } \\
\text { nha ilíaca ântero-superior, com vértice no trocânter maior do fêmur. }\end{array}$ \\
Ângulo entre o segmento de reta que passa por T12 até L5 e o segmento de reta que \\
Âssa por L5 até a espinha ilíaca póstero-inferior, com vértice em L5.
\end{tabular}

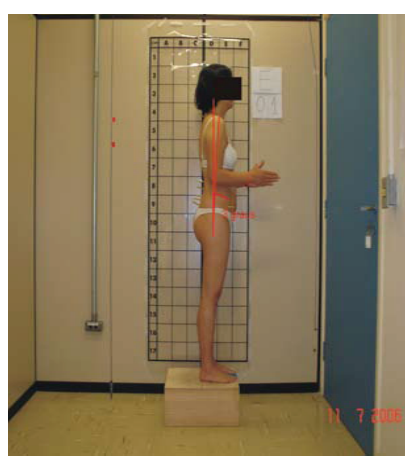

Técnica de Chaffin Modificada em ortostatismo

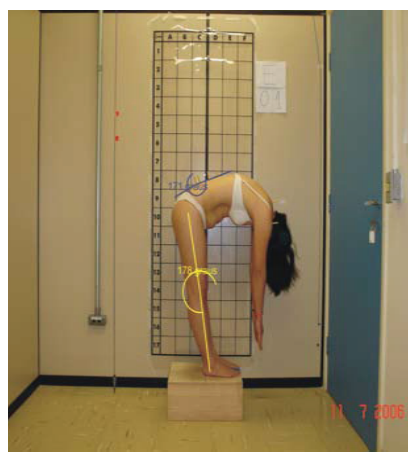

Ângulos do joelho e da lombar

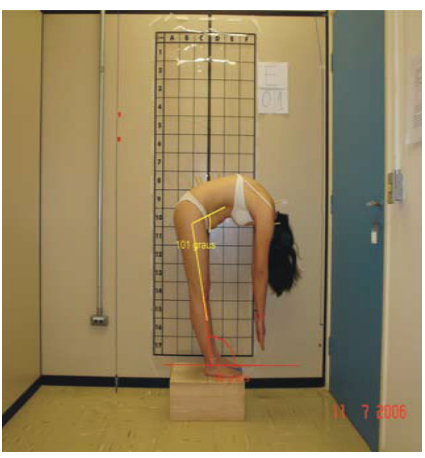

Ângulos do quadril e tíbiotársico

Figura 2. Medida dos ângulos no software Corel Draw 12.

do $\operatorname{corpo}^{21}$. Este teste avalia a flexibilidade em uma postura que se assemelha ao movimento de pegar objetos no chão, portanto, pode ser considerado mais funcional que outros testes de flexibilidade da cadeia posterior como o Banco de Wells.

No entanto, este é um teste que avalia a flexibilidade da cadeia posterior do corpo de forma global e não fornece informações sobre as articulações envolvidas na postura. Para tanto, é importante uma avaliação mais detalhada, usando, por exemplo, a fotogrametria, para se obter informações mais acuradas sobre como cada articulação está contribuindo no movimento e diagnosticar mais precisamente na qual ocorre a limitação ou ganho de flexibilidade ${ }^{17,18}$. A fotogrametria digital vem sendo considerada uma alternativa para a avaliação quantitativa de medidas lineares e angulares, possibilitando o registro da inter-relação entre partes do corpo que são difíceis de serem mensuradas por outros meios $^{19,22-25}$.Desta forma, utilizando-se a fotogrametria, foi feita a análise da Técnica de Chaffin Modificada ${ }^{19}$ e dos ângulos articulares tíbiotársico, do joelho, do quadril e da lombar por meio do software SAPo (tabela 1 e figura 2).
Após a coleta e a organização dos dados no programa Excel, as variáveis foram analisadas quanto a sua normalidade pelo teste de Shapiro Wilks e uma vez que elas apresentaram uma distribuição normal, foram feitas comparações estatísticas das variáveis obtidas entre os grupos por meio do Teste $T$,com nível de significância $\alpha=0,05$.

Para a comparação das variáveis ordinais pegar objetos no chão e tempo de permanência na posição sentada, foi utilizado o teste Qui Quadrado para comparação dos grupos. Os testes estatísticos foram realizados no programa Statistica v.7.0.

\section{RESULTADOS}

Verifica-se, primeiramente, que a idade, a estatura e a massa corporal das mulheres de ambos os grupos não são estatisticamente diferentes, confirmando a semelhança das amostras estudadas em relação às características antropométricas e de idade. Já quanto às especificidades de cada profissão, o teste do qui-quadrado mostra que há relação entre o fato das mulheres pegarem objetos no chão freqüentemente ou às vezes 
Tabela 2. Médias, desvios padrão, freqüência(\%) e valores do p das variáveis antropométricas e ocupacionais nos dois grupos estudados.

\begin{tabular}{lccc}
\hline & $\begin{array}{c}\text { Escritório } \\
(\mathrm{n}=11)\end{array}$ & $\begin{array}{c}\text { Manutenção } \\
(\mathrm{n}=13)\end{array}$ & $\mathrm{p}$ \\
\hline Idade (anos) & $36,9 \pm 12,3$ & $34,2 \pm 7,4$ & $0,5044^{1}$ \\
Estatura $(\mathrm{cm})$ & $159,7 \pm 3,1$ & $157,1 \pm 6,3$ & $0,2158^{1}$ \\
Massa corporal $(\mathrm{kg})$ & $59,5 \pm 11,5$ & $59,8 \pm 9,9$ & $0,5820^{2}$ \\
$\begin{array}{l}\text { Pegam objetos no chão freqüentemente ou às vezes } \\
\text { durante o trabalho (\%) }\end{array}$ & 27,3 & 84,6 & $0,0069^{3}$ \\
$\begin{array}{l}\text { Permanecem sentadas por mais de 3 horas durante o } \\
\text { trabalho }(\%)\end{array}$ & 81,8 & 0 & $0,0000^{3}$ \\
\hline
\end{tabular}

${ }^{1}$ Teste $t,{ }^{2}$ Teste de Mann-Whitney, ${ }^{3}$ Teste de qui-quadrado

Tabela 3. Médias, desvios padrão e valores do p das variáveis da flexibilidade e angulares avaliadas nos dois grupos.

\begin{tabular}{lccc}
\hline & Escritório $(\mathrm{n}=11)$ & Manutenção $(\mathrm{n}=13)$ & $\mathrm{p}$ \\
\hline Teste do 3o dedo ao solo (cm) & $30,2 \pm 7,0$ & $24,3 \pm 7,4$ & $0,0518^{1}$ \\
Ângulo tíbio-társico (graus) & $97,7 \pm 2,6$ & $95,8 \pm 2,0$ & $0,0609^{1}$ \\
Ângulo do joelho (graus) & $176,9 \pm 2,3$ & $175,7 \pm 2,7$ & $0,2510^{1}$ \\
Ângulo do quadril (graus) & $116,7 \pm 11,8$ & $97,2 \pm 19,0$ & $0,0713^{1}$ \\
Técnica Chaffin Modificada (graus) & $87,9 \pm 10,6$ & $99,9 \pm 11,2$ & $0,0134^{1}$ \\
Ângulo da lombar (graus) & $173,8 \pm 4,7$ & $172,0 \pm 2,8$ & $0,2523^{1}$ \\
\hline
\end{tabular}

'Teste $\mathrm{t}$

durante o trabalho e a atividade ocupacional, bem como entre esta última e o fato das mulheres permanecerem sentadas por mais de três horas durante o trabalho. Tendo essas relações confirmadas, as porcentagens demonstram que são as profissionais de manutenção que mais agacham para pegar objetos no chão, enquanto que as profissionais de escritório permanecem mais tempo sentadas (tabela 2).

As mulheres que trabalham em escritório obtiveram maiores medidas de distância do terceiro dedo ao solo em comparação às mulheres que trabalham na manutenção $(\mathrm{p}=0,0518)$. Sendo esse teste validado na literatura para mensuração da flexibilidade da cadeia posterior, pode-se confirmar que as profissionais de manutenção são mais flexíveis. Porém, não houve diferença estatística significativa na comparação dos ângulos do quadril e da lombar entre os dois grupos, o que significa que a diferença na flexibilidade observada não pode ser atribuída a essas variáveis mensuradas isoladamente. Já a técnica Chaffin Modifica$\mathrm{da}^{19}$, que soma as contribuições do quadril e de tronco inferior, assim como o ângulo tíbio-társico, mostraram-se significativamente diferentes entre os dois grupos, apresentandose capazes de diferenciar a flexibilidade entre os grupos.

O ângulo do joelho, por sua vez, foi utilizado como controle para assegurar que todas os sujeitos mantivessem os joelhos o mais próximo possível da máxima extensão, garantindo que o movimento nessa articulação não interferisse na mensuração da flexibilidade de tronco e quadril. Portanto, como era esperado, não se observou diferença estatística entre os dois grupos para essa variável (tabela 3).

\section{DISCUSSÃO}

Este estudo teve por objetivo verificar se a atividade profissional determinava alguma modificação na flexibilidade global da cadeia posterior na postura de flexão do tronco e analisar os segmentos corporais potencialmente contribuidores desta modificação.

$\mathrm{O}$ que se observou de mais evidente, no presente estudo, foi uma maior flexibilidade de tronco nas profissionais de limpeza em relação às secretárias, estabelecendo uma relação entre as atividades profissionais, dentre elas a de se curvar para apanhar objetos no solo e o aumento de flexibilidade, indicando uma provável adaptação estrutural dos tecidos corporais causada pela atividade ocupacional. Sacco et al. $(2006)^{18}$ encontraram medidas ainda menores de distância do $3^{\circ}$ dedo ao solo em profissionais de manutenção e limpeza, concordando com os achados deste estudo.

Milosavljevic et al. ${ }^{4}$ estudaram tosquiadores de ovelhas da Nova Zelândia, que mantinham 
uma flexão de tronco por longos períodos e observaram que movimentos cíclicos sagitais da coluna influenciam adaptativamente os elementos contráteis e não contráteis esqueléticos que controlam tanto a postura quanto a amplitude de movimento do tronco desses profissionais.

Os resultados apontam que as diferenças de flexibilidade entre as profissionais foram mais decorrentes de maiores contribuição dos ângulos de quadril e tibio-tarsico, considerados conjuntamente, indo ao encontro de Milosavljevic $^{4}$ que não encontrou diferença na amplitude angular na região lombar entre as ocupações estudadas.

De maneira controversa aos nossos achados e aos achados de Milosavljevic ${ }^{4}$, Torén ${ }^{24}$ não encontrou relação entre a amplitude de movimento ativa de rotação do tronco e a profissão ao comparar tratoristas, os quais realizam tal torção cíclica em suas atividades profissionais e profissionais de escritório, que não realizam esta torção. Boden e Öberg ${ }^{26}$ também não encontraram diferenças entre indivíduos destas duas profissões quanto à resistência à torção passiva do tronco e à amplitude de movimento de torção do tronco. Os autores de ambos os artigos citados anteriormente assumiram em suas discussões que esperavam tal relação entre a capacidade de movimento e a atividade profissional e desta forma, explicam esta falta de associação nos tratoristas à presença de vibração causada pelos tratores que levaria a um aumento de rigidez tecidual, impedindo as adaptações teciduais e consequentemente, funcionais.

A flexibilidade pode ser considerada um indicador de saúde corporal ${ }^{14}$, contudo, deve ser considerado que o trabalhar na posição curvada, como acontece entre os profissionais de manutenção e limpeza no presente estudo, potencialmente, leva a um aumento da extensibilidade dos tecidos passivos, como ligamentos, discos intervertebrais e tendões, o que diminuiria o torque extensor destes tecidos passivos, e solicitaria mais esforço dos tecidos ativos (músculos) durante o retorno da flexão ${ }^{27}$. A flexão cíclica, se mantida por longos períodos, leva a uma resposta inadequada por parte da musculatura extensora, o que provocaria situações potenciais de dano à região lombar ${ }^{28}$.

A flexão cíclica lombar pode desencadear uma resposta bifásica, com um aumento da flexibilidade lombar nos primeiros trinta minutos, seguido de um aumento de rigidez em alguns in- divíduos, conforme Parkinson et al. ${ }^{27}$. Para Shin e Mirka ${ }^{29}$ a resposta viscoelástica causada pela flexão prolongada pode ser moderada por intervalos de repouso entre as flexões. Esse aumento de rigidez em alguns indivíduos poderia explicar a grande variabilidade no ângulo do quadril, encontrados entre os profissionais de limpeza neste estudo. Observamos que a grande variabilidade encontrada neste ângulo entre as profissionais de limpeza, fez com que a comparação com as secretárias fosse marginalmente significativa, apesar das médias serem bastante diferentes.

Os resultados encontrados para as secretárias, por outro lado, sugerem que o trabalho na posição sentada também pode estar determinando uma adaptação estrutural dos tecidos corporais. No entanto, esta adaptação parece ser no sentido do encurtamento da cadeia posterior do corpo, já que estas profissionais se mostraram globalmente menos flexíveis.

A posição sentada coloca a musculatura posterior dos membros inferiores em encurtamento, principalmente os músculos biarticulares que atravessam o joelho como os isquiotibiais e gastrocnemios. Esta posição de encurtamento, quando mantida por longos períodos e grande freqüência, poderia levar ao encurtamento da cadeia posterior.

Além disto, a postura sentada requer menos gasto de energia e impõe menos carga sobre os membros inferiores em comparação com a posição em pé, porém, quando mantida por tempo prolongado, é normalmente associada com o aumento de forças compressivas entre L4-L5 e dores na região lombar ${ }^{30,31}$, embora haja uma recente discussão na literatura ${ }^{32,33}$ de que não há evidências científicas ou ainda há evidências contrárias à afirmação de que a postura sentada levaria a uma maior sobrecarga, degeneração discal e, conseqüentemente, dor lombar. Ainda assim, há que se considerar que as secretárias, mesmo sentadas em uma postura ideal, se esta posição for sustentada por diversas horas, pode levar à fadiga dos músculos extensores da coluna lombar ${ }^{34}$.

Portanto, a observação de intervalos de repouso adequados nos trabalhos que envolvem a flexão do tronco pode diminuir a possível morbidade associada a esta atividade ${ }^{27,29}$. Callaghan e McGillis propõem que a postura em pé seja utilizada como um descanso da postura sentada, não devendo nem a postura em pé nem a postura sentada ser mantidas isoladamente por tempo 
prolongado. Também, deve ser considerado que uma melhor utilização da amplitude fisiológica de flexão do quadril, nos movimentos funcionais de pegar objetos no chão, pode diminuir a sobrecarga na região lombar ${ }^{18}$.

\section{CONCLUSÃO}

Em mulheres, a atividade profissional influencia na flexibilidade da cadeia posterior do corpo na posição de flexão do tronco. Mulheres que trabalham predominantemente na posição sentada apresentam menor flexibilidade global da cadeia posterior quando comparadas com as que realizam um trabalho fisicamente ativo que exige flexão cíclica do tronco. A análise das amplitudes articulares dos membros inferiores e da lombar sugere que a diminuição da flexibilidade global é fruto da somatória da menor contribuição dos ângulos tíbio-társico e quadril em conjunto.

Este achado confirma a necessidade de se levar em conta o tipo de trabalho que o indivíduo realiza em programas de condicionamento físico, assim como na prevenção e reabilitação de lesões musculoesqueléticas.

A avaliação dos achados deste estudo indica que a adaptação estrutural dos tecidos corporais às atividades laborais leva a variações na amplitude de movimento. Indivíduos que trabalham predominantemente sentados possuem menos flexibilidade global da cadeia posterior em comparação com os que são mais ativos e realizam flexão cíclica do tronco. Em próximos estudos, a comparação com indivíduos que trabalham predominantemente na posição de pé ou ortostática, poderia aprofundar esta discussão.

\section{REFERÊNCIAS BIBLIOGRÁFICAS}

1. Dishman RK, Oldenburg B, O'Neal H, Shephard RJ. Worksite physical activity interventions. Am J Prev Med 1998;15(4):344-361.

2. McLean L, Tingley M, Scott RN, Rickards J. Computer terminal work and the benefit of microbreaks. Appl Ergon 2001;32(3):225-237.

3. van Dieen JH, de Looze MP, Hermans V. Effects of dynamic office chairs on trunk kinematics, trunk extensor EMG and spinal shrinkage. Ergonomics 2001;44(7)739-750.

4. Milosavljevic S, Milburn PD, Knox BW. The influence of occupation on lumbar sagittal motion and posture. Ergonomics 2005;48(6):657-667.

5. Ingraham SJ. The role of flexibility in injury prevention and athletic performance: have we stretched the truth? Minn Med 2003;86(5)58-61.
6. Dantas E. Flexibilidade: alongamento e flexionamento; Rio de Janeiro: Shape; 1999.

7. Pate RR, Pratt M, Blair SN, Haskell WL, Macera CA, Bouchard C, et al. Physical activity and public health. A recommendation from the Centers for Disease Control and Prevention and the American College of Sports Medicine. Jama 1995;273(5):402-407.

8. Kiss M. Esporte e Exercício: avaliação e Prescrição. São Paulo: Roca; 2003.

9. Jones MA, Stratton G, Reilly T, Unnithan VB. Biological risk indicators for recurrent non-specific low back pain in adolescents. Br J Sports Med 2005;39(3):137-140.

10. Wong TK, Lee RY. Effects of low back pain on the relationship between the movements of the lumbar spine and hip. Hum Mov Sci 2004;23(1)21-34.

11. Kruger J, Yore MM, Ainsworth BE, Macera CA. Is participation in occupational physical activity associated with lifestyle physical activity levels? J Occup Environ Med 2006;48(11)1143-1148.

12. Kaleta D, Makowiec-Dabrowska T, DziankowskaZaborszczyk E, Jegier A. Physical activity and selfperceived health status. Int J Occup Med Environ Health 2006;19(1):61-69.

13. Paffenbarger RS, Jr., Hale WE, Brand RJ, Hyde RT. Work-energy level, personal characteristics, and fatal heart attack: a birth-cohort effect. Am J Epidemiol 1977; 105(3):200-213.

14. Hammermeister J, Page RM, Dolny D, Burnham T. Occupational physical activity as an indicator of health and fitness. Percept Mot Skills; 2001; 92(1):121-7.

15. Ohta M, Okufuji T, Matsushima Y, Ikeda M. The effect of lifestyle modification on physical fitness and work ability in different workstyles. J Uoeh 2004; 26(4):411-421.

16. Tammelin T, Nayha S, Rintamaki H, Zitting P. Occupational physical activity is related to physical fitness in young workers. Med Sci Sports Exerc 2002;34(1): 158-165.

17. Sacco I. Avaliação de posturas sentadas em automóvel: implicações da antropometria; estudo de casos. Rev Fisioter Univ São Paulo 2003;10(1):34-42.

18. Sacco I. Influência de uma única intervenção instrutiva fisioterapêutica na flexibilidade global e amplitude angular do quadril durante a flexão do tronco. Fisioterapia e Pesquisa 2006;13(3):13-19.

19. Satto T, Vieira E, Coury H. Análise da confiabilidade de técnicas fotogramétricas para medir a flexão anterior do tronco. Rev Bras Fisioter 2003;7(1):53-59.

20. Kendall F. Músculos: provas e funções. São Paulo: Editora Manole; 1995

21. Perret C, Poiraudeau S, Fermanian J, Colau MM, Benhamou MA, Revel M. Validity, reliability, and responsiveness of the fingertip-to-floor test. Arch Phys Med Rehabil 2001;82(11):1566-1570. 
22. Watson AW, Mac Donncha C. A reliable technique for the assessment of posture: assessment criteria for aspects of posture. J Sports Med Phys Fitness 2000; 40(3):260-270.

23. Watson A. Procedure for the production of high quality photographs suitable for the recording and evaluation of posture. Rev Bras Fisioter 1998; 5(1):20-26.

24. Toren A. Muscle activity and range of motion during active trunk rotation in a sitting posture. Appl Ergon 2001;32(6):583-591.

25. Iunes D, Castro F, Salgado H, Moura I, Oliveira A, Bevilaqua-Grossi D. Confiabilidade intra e interexaminadores e repetibilidade da avaliação postural pela fotogrametria. Rev Bras Fisioter 2005;9(3):327-334.

26. Boden $\mathrm{A}$, Oberg $\mathrm{K}$. Torque resistance of the passive tissues of the trunk at axial rotation. Appl Ergon 1998;29(2):111-118.

27. Parkinson RJ, Beach TA, Callaghan JP. The timevarying response of the in vivo lumbar spine to dynamic repetitive flexion. Clin Biomech (Bristol, Avon) 2004; 19(4):330-336.

28. 28. Olson MW, Li L, Solomonow M. Flexionrelaxation response to cyclic lumbar flexion. Clin Biomech (Bristol, Avon) 2004;19(8):769-776.

29. Shin G, Mirka G. An in vivo assessment of the low back response to prolonged flexion: Interplay between active and passive tissues. Clin Biomech (Bristol, Avon) 2007.

30. Hamil J, Knutzen K. 1999, Bases Biomecânicas do Movimento Humano. São Paulo: Editora Manole.

31. Callaghan JP, McGill SM. Low back joint loading and kinematics during standing and unsupported sitting. Ergonomics; 2001;44(3):280-294.
32. Claus A, Hides J, Meseley G L, Hodges P. Sitting versus standing: Does the intradiscal pressure cause disc degeneration or low back pain?. J Electromyogr Kinesiol 2008;18(4):550-558.

33. Wilke H. New in vivo measurements of pressures in the intervertebral disc in daily life. Spine 1999; 24(8):755-762.

34. Neumann D. Cinesiologia do Aparelho Musculoesquelético. 2005, Rio de Janeiro: Guanabara Koogan.

\section{Agradecimentos}

Os autores agradecem a fundamental colaboração de Vitor Daniel Tessutti e do Colégio Marista Arquidiocesano de São Paulo na coleta dos dados.

\section{Endereço para correspondência} Isabel de C. N. Sacco.

Depto. Fonoaudiologia, Fisioterapia

e Terapia Ocupacional

Faculdade de Medicina - USP.

Rua Cipotânia, 51, Cidade Universitária 05360-160. São Paulo, SP. Brazil.

E-mail: icnsacco@usp.br 